\title{
Corrigendum: Living at the Extremes: Extremophiles and the Limits of Life in a Planetary Context
}

\author{
Nancy Merino ${ }^{1,2,3}$, Heidi S. Aronson ${ }^{4}$, Diana P. Bojanova ${ }^{1}$, Jayme Feyhl-Buska ${ }^{1}$, \\ Michael L. Wong ${ }^{5,6}$, Shu Zhang ${ }^{7}$ and Donato Giovannelli ${ }^{2,8,9,10 *}$
}

${ }^{1}$ Department of Earth Sciences, University of Southern California, Los Angeles, CA, United States, ${ }^{2}$ Earth-Life Science Institute, Tokyo Institute of Technology, Tokyo, Japan, ${ }^{3}$ Biosciences and Biotechnology Division, Physical and Life Sciences Directorate, Lawrence Livermore National Lab, Livermore, CA, United States, ${ }^{4}$ Department of Biology, University of Southern California, Los Angeles, CA, United States, ${ }^{5}$ Department of Astronomy - Astrobiology Program, University of Washington, Seattle, WA, United States, ${ }^{6}$ NASA Astrobiology Institute's Virtual Planetary Laboratory, University of Washington, Seattle, WA, United States, ${ }^{7}$ Section of Infection and Immunity, Herman Ostrow School of Dentistry of USC, University of Southern California, Los Angeles, CA, United States, ${ }^{8}$ Department of Biology, University of Naples "Federico II", Naples, Italy, ${ }^{9}$ Department of Marine and Coastal Science, Rutgers, The State University of New Jersey, New Brunswick, NJ,

United States, ${ }^{10}$ Institute for Biological Resources and Marine Biotechnology, National Research Council of Italy, Ancona, Italy

Keywords: polyextremophiles, limits of life, astrobiology, habitability and astrobiology, extremophiles/ extremophily, search for life

\section{A Corrigendum on}

Living at the Extremes: Extremophiles and the Limits of Life in a Planetary Context by Merino, N., Aronson, H. S., Bojanova, D. P., Feyhl-Buska, J., Wong, M. L., Zhang, S., et al. (2019) Front. Microbiol. 10:780. doi: 10.3389/fmicb.2019.00780

\section{OPEN ACCESS}

Edited and reviewed by: Davide Zannoni,

University of Bologna, Italy

*Correspondence:

Donato Giovannelli donato.giovannelli@unina.it

Specialty section: This article was submitted to Extreme Microbiology, a section of the journal

Frontiers in Microbiology

Received: 02 July 2019

Accepted: 18 July 2019

Published: 13 August 2019

Citation:

Merino N, Aronson HS, Bojanova DP, Feyhl-Buska J, Wong ML, Zhang S

and Giovannelli D (2019)

Corrigendum: Living at the Extremes: Extremophiles and the Limits of Life in a Planetary Context.

Front. Microbiol. 10:1785

doi: 10.3389/fmicb.2019.01785
In the original article, there was a mistake in the legend for Table 4 as published. The legend in Table 4 is missing two parentheses around "Poly." The correct legend appears below.

"Table 4. Examples of notable (Poly)extremophiles and their physiological requirements."

Additionally, there was a mistake in Table 3 and Table 5 as published. In Table 3, the lowest temperature listed for Planococcus halocryophilus Or1 is " $-18^{\circ} \mathrm{C}$." It should be " $-15^{\circ} \mathrm{C}^{\prime}$ instead. In addition, the $\mathrm{pH}$ range is " $\mathrm{nr}$ " but should be " $6-11$ " instead. In the temperature column, 37 is bold type, but this should be regular type.

In Table 5, the atmosphere entry for Earth > Atmosphere > Geochemistry is listed as " $8.1 \%$ $\mathrm{N}_{2}$," but the actual composition of Earth's atmosphere is " $78 \% \mathrm{~N}_{2}$."

The corrected Table 3 and Table 5 appear below.

Lastly, there is a grammatical error in the original article.

A correction has therefore been made to the section Can Life Originate, Evolve, or Survive on Other Planetary Bodies?, paragraph five:

"Solar and galactic cosmic rays (high-energy particles with energies from $10 \mathrm{MeV}$ to $>10$ $\mathrm{GeV}$ ) present challenges to life on the surface and near-surface of Mars and other planetary bodies. However, any subsurface aquifer deeper than a few meters would be protected from damaging radiation. Dartnell et al. (2007) calculated the galactic cosmic ray dosage rates and the corresponding survival times (which they defined as a million-fold decrease in cell number) of characteristic microbes at different depths in Mars's subsurface. At the surface, E. coli has a survival time of 1,200 years, while at 20 -m depth, that survival time jumps to $1.5 \times 10^{8}$ years. Compared to E. coli, D. radiodurans has survival times an order of magnitude longer. These survival times are, in fact, lower limits in light of recent measurements by the Radiation Assessment Detector onboard the Mars Science Laboratory (Hassler et al., 2014), which found that the actual dose rate at Gale Crater (76 $\mathrm{mGy}_{\text {year }}{ }^{-1}$ ) is a factor of 2 lower than that modeled by Dartnell et al. (2007)."

The authors apologize for these errors and state that they do not change the scientific conclusions of the article in any way. The original article has been updated. 
TABLE 3 | Limits of life as identified by (poly)extremophilic organisms in pure cultures.

\begin{tabular}{|c|c|c|c|c|c|c|c|c|c|}
\hline Strain & Domain & $\begin{array}{l}\text { Extremophile } \\
\text { Type }\end{array}$ & $\begin{array}{l}\text { Isolation } \\
\text { ecosystem }\end{array}$ & $\begin{array}{c}\text { Temperature } \\
\left({ }^{\circ} \mathrm{C}\right)\end{array}$ & $\mathrm{pH}$ & $\begin{array}{l}\text { Pressure } \\
\text { (Mpa) }\end{array}$ & Salinity (\%) & $\begin{array}{c}\text { Water } \\
\text { activity }\left(a_{w}\right)\end{array}$ & References \\
\hline $\begin{array}{l}\text { Picrophilus } \\
\text { oshimae KAW 2/2 }\end{array}$ & Archaea & Hypercidophile & $\begin{array}{l}\text { Hot springs, } \\
\text { Solfataras }\end{array}$ & $47-65(60)^{a}$ & $\begin{array}{c}-\mathbf{0 . 0 6 - 1 . 8} \\
(0.7)\end{array}$ & $\mathrm{nr}$ & $0-20$ & $\mathrm{nr}$ & $\begin{array}{l}\text { Schleper et al., } \\
\text { 1995, } 1996\end{array}$ \\
\hline $\begin{array}{l}\text { Serpentinomonas } \\
\text { sp. B1 }\end{array}$ & Bacteria & Alkaliphile & $\begin{array}{l}\text { Serpentinizing } \\
\text { system } \\
\text { (water) }\end{array}$ & 18-37 (30) & $9-12.5(11)$ & $\mathrm{nr}$ & $0-0.5(0)$ & $\mathrm{nr}$ & Suzuki et al., 2014 \\
\hline $\begin{array}{l}\text { Methanopyrus } \\
\text { kandleri } 116\end{array}$ & Archaea & Hyperthemophile & $\begin{array}{l}\text { Deep-sea } \\
\text { hydrothermal } \\
\text { vent }\end{array}$ & 90-122 (105) & (6.3-6.6) & $0.4-40$ & $0.5-4.5(3.0)$ & $\mathrm{nr}$ & Takai et al., 2008 \\
\hline $\begin{array}{l}\text { Planococcus } \\
\text { halocryophilus Or1 }\end{array}$ & Bacteria & Halopsychrophile & Sea ice core & -15-37 (25) & $6-11(7-8)$ & $\mathrm{nr}$ & $0-19(2)$ & $\mathrm{nr}$ & $\begin{array}{l}\text { Mykytczuk et al., } \\
2012,2013\end{array}$ \\
\hline $\begin{array}{l}\text { Halarsenatibacter } \\
\text { silvermanii SLAS-1 }\end{array}$ & Bacteria & Haloalkaliphile & Soda lake & $28-55(44)$ & $8.7-9.8(9.4)$ & $\mathrm{nr}$ & 20-35 (35) & $\mathrm{nr}$ & $\begin{array}{l}\text { Oremland et al., } \\
2005\end{array}$ \\
\hline $\begin{array}{l}\text { Thermococcus } \\
\text { piezophilus CDGS }\end{array}$ & Archaea & Piezothermophile & $\begin{array}{l}\text { Deep-sea } \\
\text { hydrothermal } \\
\text { vent }\end{array}$ & 60-95 (75) & $5.5-9(6)$ & $0.1-125(50)$ & 2-6 (3) & $\mathrm{nr}$ & $\begin{array}{l}\text { Dalmasso et al., } \\
2016\end{array}$ \\
\hline $\begin{array}{l}\text { Haloarchaeal } \\
\text { strains GN-2 and } \\
\text { GN-5 }\end{array}$ & Archaea & Xerophile & $\begin{array}{l}\text { Solar salterns } \\
\text { (brine) }\end{array}$ & $\mathrm{nr}$ & $\mathrm{nr}$ & $\mathrm{nr}$ & $\mathrm{nr}$ & 0.635 & Javor, 1984 \\
\hline
\end{tabular}

${ }^{a}$ Data presented as range (optimum) for each parameter. nr, not reported in the original publication. Current limits are highlighted in bold. 
TABLE 5 | Boundary conditions for different planetary bodies of astrobiological interest (compared to Earth), split into atmosphere, surface, and subsurface layers.

\begin{tabular}{|c|c|c|c|c|c|c|c|c|}
\hline $\begin{array}{l}\text { Planetary } \\
\text { body }\end{array}$ & Type & Layer & $\begin{array}{l}\text { Temperature } \\
\left({ }^{\circ} \mathrm{C}\right)\end{array}$ & $\mathrm{pH}$ & Pressure (MPa) & Salinity (\% $\mathrm{NaCl})$ & Geochemistry & References \\
\hline \multirow[t]{3}{*}{ Earth } & Planet & Atmosphere & $-100-40$ & $\begin{array}{l}\text { Neutral, local } \\
\text { acidic conditions } \\
\text { possible due to } \\
\text { volcanism and } \\
\text { human activities }\end{array}$ & $0.0001-0.1$ & 0 & 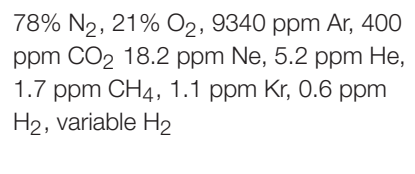 & $\begin{array}{l}\text { Hans Wedepohl, 1995; McDonough } \\
\text { and Sun, 1995; Wayne, } 2000\end{array}$ \\
\hline & & Surface & $-98.6-464$ & $-3.6-13.3$ & $0.003-112$ & 0 - saturation & $\begin{array}{l}\text { Soils and sediments of varying } \\
\text { lithologies, siliceous crust, ranging } \\
\text { from mafic to felsic composition. } \\
\text { Extensive ocean ( } 70 \% \text { planet } \\
\text { surface), with } 4,000 \mathrm{~m} \text { average depth, } \\
4^{\circ} \mathrm{C} \text { and } 3.5 \% \text { average temperature } \\
\text { and salinity respectively }\end{array}$ & \\
\hline & & Subsurface & $3.25-<400$ & $\sim 1-12.8$ & $<800$ & 0.05 - saturation & $\begin{array}{l}\text { Soils and sediments of varying } \\
\text { lithologies, siliceous crust, ranging } \\
\text { from mafic to felsic composition, } \\
\text { ultramafic mantle }\end{array}$ & \\
\hline \multirow[t]{3}{*}{ Venus } & Planet & Atmosphere & $-40-482^{a}$ & $0^{b}$ & $0.1-9.3^{c}$ & $\mathrm{nr}$ & $\begin{array}{l}96.5 \% \mathrm{CO}_{2}, 3.5 \% \mathrm{~N}_{2} ; \text { small } \\
\text { quantities of } \mathrm{CO}, \mathrm{SO}_{2}, \mathrm{HCl}, \mathrm{HF}, \mathrm{HDO} \text {, } \\
\text { and } \mathrm{H}_{2} \mathrm{O} ; \mathrm{H}_{2} \mathrm{SO}_{4} \text { condensates }\end{array}$ & $\begin{array}{l}\text { Cockell, 1999; Basilevsky and Head, } \\
\text { 2003; Schulze-Makuch et al., 2004; } \\
\text { Lang and Hansen, 2006; Bertaux } \\
\text { et al., 2007; Airey et al., } 2017\end{array}$ \\
\hline & & Surface & $377-482$ & $\mathrm{nr}$ & $4.5-9.3^{C}$ & $\mathrm{nr}$ & $\begin{array}{l}\text { Rocks are similar to tholeiitic and } \\
\text { alkaline basalts; no liquid water }\end{array}$ & \\
\hline & & Subsurface & $\mathrm{nr}$ & $\mathrm{nr}$ & $\mathrm{nr}$ & $\mathrm{nr}$ & Fluid channels; volcanism & \\
\hline \multirow[t]{3}{*}{ Mars } & Planet & Atmosphere & $-138-35^{d}$ & $\mathrm{nr}$ & $0.0001-0.0009$ & $\mathrm{nr}$ & $\begin{array}{l}\text { 95. 3\% } \mathrm{CO}_{2}, 2.7 \% \mathrm{~N}_{2}, 1.6 \% \mathrm{Ar} \text {, } \\
0.13 \% \mathrm{O}_{2}, 0.08 \% \mathrm{CO} \text {; trace amounts } \\
\text { of } \mathrm{H}_{2} \mathrm{O}, \mathrm{NO}, \mathrm{Ne}, \mathrm{Kr} \text {, Xe }\end{array}$ & $\begin{array}{l}\text { Varnes et al., 2003; Fairén et al., } \\
\text { 2004; Nicholson and Schuerger, } \\
\text { 2005; Hecht et al., 2009; Smith et al., } \\
\text { 2009; Johnson et al., 2011; Jones } \\
\text { et al., 2011; Michalski et al., 2013; } \\
\text { Longstaff, 2014; Wordsworth, 2016; } \\
\text { Sinha et al., 2017; NASA, } 2018\end{array}$ \\
\hline & & Surface & $-138-30$ & $7.7^{\mathrm{e}}$ & $0.0004-0.0009$ & $5.2-5.8$ & $\begin{array}{l}\text { Basaltic, Fe-/Mg-rich phyllosilicates, } \\
\text { perchlorate salts, Al-rich clays, } \\
\text { sulfates, chlorides, calcite, and silicas; } \\
\text { potential cryosphere }\end{array}$ & \\
\hline & & Subsurface & 559 & $4.96-9.13^{h}$ & $10-3039$ & Cl-rich brines & $\begin{array}{l}\text { Potential groundwater; basalt crust; } \\
\text { possible serpentinization }\end{array}$ & \\
\hline
\end{tabular}


TABLE 5 | Continued

\begin{tabular}{|c|c|c|c|c|c|c|c|c|}
\hline $\begin{array}{l}\text { Planetary } \\
\text { body }\end{array}$ & Type & Layer & $\begin{array}{l}\text { Temperature } \\
\left({ }^{\circ} \mathrm{C}\right)\end{array}$ & $\mathrm{pH}$ & Pressure (MPa) & Salinity (\% NaCl) & Geochemistry & References \\
\hline \multirow[t]{3}{*}{ Enceladus } & lcy moon & Plume jets & 0 & $\sim 8.5-9$ & High velocity jets & $>0.5$ & $\begin{array}{l}90-99 \% \mathrm{H}_{2} \mathrm{O}, \leq 0.61-4.27 \% \mathrm{~N}_{2} \text {, } \\
0.3-5.3 \% \mathrm{CO}_{2}, 0.1-1.68 \% \mathrm{CH}_{4} \text {, } \\
\text { 0.4-0.9\% } \mathrm{NH}_{3}, 0.4-39 \% \mathrm{H}_{2} \text {, trace } \\
\text { amounts of hydrocarbons; high mass } \\
\text { organic cations, silicates, sodium, } \\
\text { potassium, carbonates }\end{array}$ & $\begin{array}{l}\text { Gioia et al., 2007; Postberg et al., } \\
\text { 2009, 2018; Waite et al., 2009; } \\
\text { Zolotov et al., 2011; Glein et al., } \\
\text { 2015; Holm et al., 2015; Hsu et al., } \\
\text { 2015; Taubner et al., } 2018\end{array}$ \\
\hline & & $\begin{array}{l}\text { lcy shell } \\
\text { ( 10 km thick) }\end{array}$ & $-233--23$ & $\mathrm{nr}$ & $\mathrm{nr}$ & $\begin{array}{l}\text { May have } \\
\text { ammonia brine } \\
\text { pockets }\end{array}$ & May have tectonics & \\
\hline & & $\begin{array}{l}\text { Subsurface } \\
\text { global ocean } \\
(\sim 0-170 \mathrm{~km} \\
\text { depth) }\end{array}$ & $<90$ & $8.5-12.2^{k}$ & $1-8$ & $0.45-<4$ & Possible serpentinization & \\
\hline \multirow[t]{3}{*}{ Titan } & lcy moon & Atmosphere & $-183--73^{j}$ & $\mathrm{nr}$ & $>0.01-0.15$ & $\mathrm{nr}$ & $\begin{array}{l}98.4 \% \mathrm{~N}_{2}, 1.4 \% \mathrm{CH}_{4}, 0.2 \% \mathrm{H}_{2} \\
\text { trace hydrocarbons and organics; } \\
95 \% \mathrm{~N}_{2}, 5 \% \mathrm{CH}_{4}, 0.1 \% \mathrm{H}_{2} ; \sim 50 \\
\text { ppmv CO and } \sim 15 \text { ppbv } \mathrm{CO}_{2} ; \\
\mathrm{C}_{2} \mathrm{H}_{3} \mathrm{CN} \text {; clouds }\end{array}$ & $\begin{array}{l}\text { Fulchignoni et al., 2005; de Kok et al., } \\
\text { 2007; Norman, 2011; Baland et al., } \\
\text { 2014; Mastrogiuseppe et al., 2014; } \\
\text { Mitri et al., 2014; Sohl et al., 2014; } \\
\text { Jennings et al., 2016; McKay, 2016; } \\
\text { Mitchell and Lora, 2016; Brassé et al., } \\
\text { 2017; Cordier et al., } 2017\end{array}$ \\
\hline & & Surface & $-183--179$ & $\mathrm{nr}$ & $0.15-0.35^{i}$ & $\mathrm{nr}$ & $\begin{array}{l}\text { Lakes and sea have } \mathrm{CH}_{4}, \mathrm{C}_{2} \mathrm{H}_{4} \text {, and } \\
\text { dissolved nitrogen; dunes of solid } \\
\text { organic material; low-latitude deserts } \\
\text { and high-latitude moist climates }\end{array}$ & \\
\hline & & Subsurface & -18 & $11.8^{1}$ & $50-300^{m}$ & $\begin{array}{l}\text { Likely dense } \\
\text { subsurface ocean } \\
\left(\leq 1,350 \mathrm{~kg} \mathrm{~m}^{-3}\right) \\
\text { suggesting high } \\
\text { salinity }\end{array}$ & $\mathrm{CH}_{4}$ and $\mathrm{C}_{2} \mathrm{H}_{6}$ & \\
\hline Ceres & $\begin{array}{l}\text { Dwarf } \\
\text { planet }\end{array}$ & Atmosphere & $\mathrm{nr}$ & $\mathrm{nr}$ & $\mathrm{nr}$ & $\mathrm{nr}$ & $\begin{array}{l}\text { Transient atmosphere with possible } \\
\text { water vapo }\end{array}$ & $\begin{array}{l}\text { Fanale and Salvail, 1989; Zolotov, } \\
\text { 2009, 2017; Küppers et al., 2014; } \\
\text { Hayne and Aharonson, 2015; Neveu } \\
\text { and Desch, 2015; Hendrix et al., } \\
\text { 2016; Villarreal et al., 2017; Vu et al., } \\
\text { 2017; Castillo-Rogez et al., 2018; } \\
\text { McCord and Castillo-Rogez, 2018; } \\
\text { McCord and Zambon, } 2019\end{array}$ \\
\hline
\end{tabular}




\begin{tabular}{|c|c|c|c|c|c|c|c|c|}
\hline $\begin{array}{l}\text { Planetary } \\
\text { body }\end{array}$ & Type & Layer & $\begin{array}{l}\text { Temperature } \\
\left({ }^{\circ} \mathrm{C}\right)\end{array}$ & $\mathrm{pH}$ & Pressure (MPa) & Salinity (\% $\mathrm{NaCl})$ & Geochemistry & References \\
\hline & & Surface & $(-157--30)^{\mathrm{n}}$ & $9.7-11.3^{n}$ & $\mathrm{nr}$ & $<10^{n}$ & $\begin{array}{l}\text { Surface clays; (Mg, Ca)-carbonates; } \\
\text { (Mg, NH} 4 \text { )-phyllosilicates; Fe-rich } \\
\text { clays; salt deposits; chloride salts; } \\
\text { water-rock interactions; brucite and } \\
\text { magnetite; sulfur species and } \\
\text { graphitized carbon; localized } \\
\left.\mathrm{Na}^{-} \text {-carbonates (e.g., } \mathrm{Na}_{2} \mathrm{CO}_{3}\right) \\
\mathrm{NH}_{4} \mathrm{Cl}, \mathrm{NH}_{4} \mathrm{HCO}_{3}\end{array}$ & \\
\hline & & Subsurface & $-143--93^{\circ}$ & Likely alkaline & $<140-200^{p}$ & $\begin{array}{l}\text { Potentially has } \\
\text { briny or } \mathrm{NH}_{3} \text {-rich } \\
\text { subsurface liquid }\end{array}$ & $\begin{array}{l}\text { Active water/ice-driven subsurface } \\
\text { processes }\end{array}$ & \\
\hline \multirow[t]{3}{*}{ Europa } & Icy moon & $\begin{array}{l}\text { Atmosphere } \\
\text { (tenuous) }\end{array}$ & $\mathrm{nr}$ & $\mathrm{nr}$ & $0.1^{-12}-1^{-12}$ & $\mathrm{nr}$ & $\begin{array}{l}\text { lon sputtering of the surface; potential } \\
\text { water plumes; } \mathrm{O}_{2} \text {; trace amounts of } \\
\text { sodium and potassium }\end{array}$ & $\begin{array}{l}\text { Spencer et al., 1999; Chyba and } \\
\text { Phillips, 2001; Marion et al., 2005; } \\
\text { McGrath et al., 2009; Zolotov and } \\
\text { Kargel, 2009; Travis et al., 2012; } \\
\text { Cassidy et al., 2013; Muñoz-Iglesias } \\
\text { et al., 2013; Kattenhorn and Prockter, } \\
\text { 2014; Soderlund et al., 2014; Hand } \\
\text { and Carlson, 2015; Kimura and } \\
\text { Kitadai, 2015; Noell et al., 2015; } \\
\text { Vance et al., 2016; Teolis et al., 2017; } \\
\text { Zhu et al., 2017; Jones et al., 2018; } \\
\text { Martin and McMinn, 2018; Pavlov } \\
\text { et al., } 2018\end{array}$ \\
\hline & & $\begin{array}{l}\text { Surface (icy } \\
\text { shell) }\end{array}$ & $-187--141$ & $\mathrm{nr}$ & $0.1^{-12}$ & $\begin{array}{l}\text { May be saline, as } \\
\text { delivered to the } \\
\text { surface from a } \\
\text { salty ocean, may } \\
\text { have brine or salt } \\
\text { inclusions }\end{array}$ & $\begin{array}{l}\mathrm{H}_{2} \mathrm{O}_{2}, \mathrm{H}_{2} \mathrm{SO}_{4}, \mathrm{CO}_{2} \text {; salts } \\
\text { concentrated in cracks; oxidants and } \\
\text { simple organics; potentially } \mathrm{MgSO}_{4} \text {, } \\
\mathrm{Na}_{2} \mathrm{SO}_{4}, \mathrm{Na}_{2} \mathrm{CO}_{3} \text {, may have gas } \\
\text { inclusions; may have tectonics }\end{array}$ & \\
\hline & & $\begin{array}{l}\text { Subsurface } \\
\text { ocean }\end{array}$ & $\begin{array}{l}\text { Daily inundation } \\
\text { of seawater at } \\
T=-4-0\end{array}$ & $\begin{array}{l}\text { Potential for } \\
\text { wide range }\end{array}$ & $0.1-30^{r}$ & $<3.5$ & $\begin{array}{l}\text { Likely contains } \mathrm{Mg}^{2+}, \mathrm{SO}_{4}^{2-}, \mathrm{Na}^{+} \text {, } \\
\mathrm{Cl}^{-} \text {; oxidants and simple organics }\end{array}$ & \\
\hline
\end{tabular}

The observed or putative geochemistry as well as other potential influences are also listed. ${ }^{a}$ Thermosphere can be as cold as $-173^{\circ} \mathrm{C}$ (Bertaux et al., 2007); the upper-to-middle cloud layers are between -40 and $60^{\circ} \mathrm{C}$ (Cockell, 1999). ${ }^{b}$ Acid concentration in upper cloud layer is $81 \%$, in lower layers up to $98 \%$ (Cockell, 1999). 'C Up to 11 MPa in a deep depression (Basilevsky and Head, 2003) d Summer air temperatures on Mars near the equator can reach a maximum of $35^{\circ} \mathrm{C}$ (Longstaff, 2014). ${ }^{e}$ Measured by the Phoenix Mars Lander Wet Chemistry Laboratory at the northern plains of the Vastitas Borealis (Hecht et al., 2009). ${ }^{f}$ Liquid water may have had water activity $>0.95$ (Fairén et al., 2009). ${ }^{g}$ Calculated temperature at a depth of 1-30 km (Jones et al., 2011; Sinha et al., 2017); at a depth $\sim 310 \mathrm{~km}$, the calculated temperature is $<427^{\circ} \mathrm{C}$ (Jones et al., 2011); the Martian core has temperature 1527 ${ }^{\circ} \mathrm{C}($ Longstaff, 2014). ${ }^{h}$ Calculated groundwater pH (Narnes et al., 2003). ' ${ }^{~ C}$ Calculated pressure at Titan's large sea, Ligeia Mare, is 0.20-0.35 MPa (Cordier et al., 2017). ${ }^{j}$ Tropospheric temperature can be - 193 ${ }^{\circ} \mathrm{C}$; 80\% of incident sunlight is absorbed by Titan's atmosphere, suggesting that there are greenhouse and antigreenhouse effects (Mitchell and Lora, 2016). ${ }^{k}$ The subsurface ocean on Enceladus could also have pH range 10.8-13.5 (Glein et al., 2015). 'Calculated ocean pH with 5 wt\% NH (Brassé et al,, 2017). ${ }^{m}$ Calculated pressure for the subsurface ocean with thickness $100 \mathrm{~km}$ and outer shell thickness 40-170 km (Baland et al., 2014); 800 MPa at the mantle ice shell-core boundary (Sohl et al., 2014). ${ }^{n}$ Calculated surface temperatures, illuminated surfaces can have temperature $<-173^{\circ} \mathrm{C}$ (Hayne and Aharonson, 2015); calculated pH and salinity for bright deposits in Occator crater (Zolotov, 2017); temperature for bright deposits in Occator crater might reach $<-0.2^{\circ} \mathrm{C}$ (Zolotov, 2017). Internal temperature might reach $77^{\circ} \mathrm{C}$ (McCord and Sotin, 2005). ${ }^{p}$ Ceres' center pressure (Zolotov, 2009). ${ }^{9}$ Acid brine may result from hydrothermal systems and be enriched with sulfuric acid (Kargel et al., 2000); neutral brine may occur as leachate from chondritic material and be enriched with magnesium sulfate (Kargel et al., 2000; Pasek and Greenberg. 2012): alkaline brine may occur in areas with natron $\left(\mathrm{Na}_{2} \mathrm{CO}_{3} 10 \mathrm{H}_{2} \mathrm{O}\right.$ ), produced from the venting of $\mathrm{CO}_{2}$ from aqueous reservoirs (Langmuir, 1971; Millero and Rabindra, 1997). ' $\mathrm{At}$ the base of a $100 \mathrm{~km}$ Europan ocean, the pressure is calculated to be 146 MPa (Marion et al., 2005). 


\section{REFERENCES}

Airey, M. W., Mather, T. A., Pyle, D. M., and Ghail, R. C. (2017). The distribution of volcanism in the Beta-Atla-Themis region of Venus: its relationship to rifting and implications for global tectonic regimes. J. Geophys. Res. Planets 122, 1626-1649. doi: 10.1002/2016JE005205

Baland, R. M., Tobie, G., Lefèvre, A., and Van Hoolst, T. (2014). Titan's internal structure inferred from its gravity field, shape, and rotation state. Icarus 237, 29-41. doi: 10.1016/j.icarus.2014.04.007

Basilevsky, A. T., and Head, J. W. (2003). The surface of Venus. Rep. Prog. Phys. 66, 1699-1734. doi: 10.1088/0034-4885/66/10/R04

Bertaux, J.-L., Vandaele, A.-C., Korablev, O., Villard, E., Fedorova, A., Fussen, D., et al. (2007). A warm layer in Venus' cryosphere and highaltitude measurements of $\mathrm{HF}, \mathrm{HCl}, \mathrm{H} 2 \mathrm{O}$ and HDO. Nature 450, 646-649. doi: 10.1038/nature05974

Brassé, C., Buch, A., Coll, P., and Raulin, F. (2017). Low-temperature alkaline pH hydrolysis of oxygen-free Titan Tholins: carbonates' Impact. Astrobiology 17, 8-26. doi: 10.1089/ast.2016.1524

Cassidy, T. A., Paranicas, C. P., Shirley, J. H., Dalton, J. B., Teolis, B. D., Johnson, R. E., et al. (2013). Magnetospheric ion sputtering and water ice grain size at Europa. Planet. Space Sci. 77, 64-73. doi: 10.1016/j.pss.2012.07.008

Castillo-Rogez, J., Neveu, M., McSween, H. Y., Fu, R. R., Toplis, M. J., and Prettyman, T. (2018). Insights into Ceres's evolution from surface composition. Meteorit. Planet. Sci. 53, 1820-1843. doi: 10.1111/maps.13181

Chyba, C., and Phillips, C. (2001). Possible ecosystems and the search for life on Europa. Proc. Natl. Acad. Sci. U.S.A. 98, 801-804. doi: 10.1073/pnas.98.3.801

Cockell, C. S. (1999). Life on Venus. Planet. Space Sci. 47, 1487-1501. doi: 10.1016/S0032-0633(99)00036-7

Cordier, D., Garciá-Sánchez, F., Justo-Garciá, D. N., and Liger-Belair, G. (2017). Bubble streams in Titan's seas as a product of liquid N2 + CH4 + C2H6 cryogenic mixture. Nat. Astron. 1:0102. doi: 10.1038/s41550-0170102

Dalmasso, C., Oger, P., Selva, G., Courtine, D., L'Haridon, S., Garlaschelli, A., et al. (2016). Thermococcus piezophilus sp. nov., a novel hyperthermophilic and piezophilic archaeon with a broad pressure range for growth, isolated from a deepest hydrothermal vent at the Mid-Cayman Rise. Syst. Appl. Microbiol. 39, 440-444. doi: 10.1016/j.syapm.2016.08.003

Dartnell, L. R., Desorgher, L., Ward, J. M., and Coates, A. J. (2007). Modelling the surface and subsurface Martian radiation environment: implications for astrobiology. Geophys. Res. Lett. 34, 4-9. doi: 10.1029/2006GL027494

de Kok, R., Irwin, P. G. J., Teanby, N. A., Lellouch, E., Bézard, B., Vinatier, S., et al. (2007). Oxygen compounds in Titan's stratosphere as observed by Cassini CIRS. Icarus 186, 354-363. doi: 10.1016/J.ICARUS.2006.09.016

Fairén, A. G., Davila, A. F., Gago-Duport, L., Amils, R., and McKay, C. P. (2009). Stability against freezing of aqueous solutions on early Mars. Nature 459, 401-404. doi: 10.1038/nature07978

Fairén, A. G., Fern?ndez-Remolar, D., Dohm, J. M., Baker, V. R., and Amils, R. (2004). Inhibition of carbonate synthesis in acidic oceans on early Mars. Nature 431, 423-426. doi: 10.1038/nature02911

Fanale, F. P., and Salvail, J. R. (1989). The water regime of asteroid (1) Ceres. Icarus 82, 97-110. doi: 10.1016/0019-1035(89)90026-2

Fulchignoni, M., Ferri, F., Angrilli, F., Ball, A. J., Bar-Nun, A., Barucci, M. A., et al. (2005). In situ measurements of the physical characteristics of Titan's environment. Nature 438, 785-791. doi: 10.1038/nature04314

Gioia, G., Chakraborty, P., Marshak, S., and Kieffer, S. W. (2007). Unified model of tectonics and heat transport in a frigid Enceladus. Proc. Natl. Acad. Sci. U.S.A. 104, 13578-13581. doi: 10.1073/pnas.0706018104

Glein, C. R., Baross, J. A., and Waite, J. H. (2015). The pH of Enceladus' ocean. Geochim. Cosmochim. Acta 162, 202-219. doi: 10.1016/j.gca.2015.04.017

Hand, K. P., and Carlson, R. W. (2015). Europa's surface color suggests an ocean rich with sodium chloride. Geophys. Res. Lett. 42, 3174-3178. doi: 10.1002/2015GL063559

Hans Wedepohl, K. (1995). The composition of the continental crust. Geochim. Cosmochim. Acta 59, 1217-1232. doi: 10.1016/0016-7037(95)00038-2

Hassler, D. M., Zeitlin, C., Wimmer-schweingruber, R. F., Ehresmann, B., Rafkin, S., Eigenbrode, J. L., et al. (2014). Mars' surface radiation environment. Science 343:1244797. doi: 10.1126/science.1244797
Hayne, P. O., and Aharonson, O. (2015). Thermal stability of ice on Ceres with rough topography. J. Geophys. Res. E Planets 120, 1567-1584. doi: 10.1002/2015JE004887

Hecht, M. H., Kounaves, S. P., Quinn, R. C., West, S. J., Young, S. M. M., Ming, D. W., et al. (2009). Detection of perchlorate and the soluble chemistry of Martian soil at the phoenix lander site. Science 325, 64-67. doi: 10.1126/science.1172466

Hendrix, A. R., Vilas, F., and Li, J. Y. (2016). Ceres: sulfur deposits and graphitized carbon. Geophys. Res. Lett. 43, 8920-8927. doi: 10.1002/2016GL070240

Holm, N. G., Oze, C., Mousis, O., Waite, J. H., and Guilbert-Lepoutre, A. (2015). Serpentinization and the Formation of $\mathrm{H} 2$ and $\mathrm{CH} 4$ on Celestial Bodies (Planets, Moons, Comets). Astrobiology 15, 587-600. doi: $10.1089 /$ ast.2014.1188

Hsu, H. W., Postberg, F., Sekine, Y., Shibuya, T., Kempf, S., Horányi, M., et al. (2015). Ongoing hydrothermal activities within Enceladus. Nature 519, 207-210. doi: 10.1038/nature14262

Javor, B. (1984). Growth potential of halophilic bacteria isolated from solar salt environments: carbon sources and salt requirements. Appl. Environ. Microbiol. $48,352-360$.

Jennings, D. E., Cottini, V., Nixon, C. A., Achterberg, R. K., Flasar, F. M., Kunde, V. G., et al. (2016). Surface temperatures on Titan during northern winter and spring. Astrophys. J. 816:L17. doi: 10.3847/2041-8205/816/1/L17

Johnson, A. P., Pratt, L. M., Vishnivetskaya, T., Pfiffner, S., Bryan, R. A., Dadachova, E., et al. (2011). Extended survival of several organisms and amino acids under simulated martian surface conditions. Icarus 211, 1162-1178. doi: 10.1016/j.icarus.2010.11.011

Jones, E. G., Lineweaver, C. H., and Clarke, J. D. (2011). An extensive phase space for the potential martian biosphere. Astrobiology 11, 1017-1033. doi: $10.1089 /$ ast.2011.0660

Jones, R. M., Goordial, J. M., and Orcutt, B. N. (2018). Low energy subsurface environments as extraterrestrial analogs. Front. Microbiol. 9:1605. doi: $10.3389 /$ fmicb. 2018.01605

Kargel, J. S., Kaye, J. Z., Head, J. W., Marion, G. M., Sassen, R., Crowley, J. K., et al. (2000). Europa?s crust and ocean: origin, composition, and the prospects for life. Icarus 148, 226-265. doi: 10.1006/ICAR.2000.6471

Kattenhorn, S. A., and Prockter, L. M. (2014). Evidence for subduction in the ice shell of Europa. Nat. Geosci. 7, 762-767. doi: 10.1038/ngeo2245

Kimura, J., and Kitadai, N. (2015). Polymerization of building blocks of life on Europa and other icy moons. Astrobiology 15, 430-441. doi: 10.1089/ast.2015.1306

Küppers, M., O’Rourke, L., Bockelée-Morvan, D., Zakharov, V., Lee, S., Von Allmen, P., et al. (2014). Localized sources of water vapour on the dwarf planet (1) Ceres. Nature 505, 525-527. doi: 10.1038/nature12918

Lang, N. P., and Hansen, V. L. (2006). Venusian channel formation as a subsurface process. J. Geophys. Res. E Planets 111:E04001. doi: 10.1029/2005JE002629

Langmuir, D. (1971). The geochemistry of some carbonate ground waters in central Pennsylvania. Geochim. Cosmochim. Acta 35, 1023-1045. doi: 10.1016/0016-7037(71)90019-6

Longstaff, A. (2014). Astrobiology: An Introduction. Boca Raton, FL: CRC Press. doi: $10.1201 / \mathrm{b} 17880$

Marion, G. M., Kargel, J. S., Catling, D. C., and Jakubowski, S. D. (2005). Effects of pressure on aqueous chemical equilibria at subzero temperatures with applications to Europa. Geochim. Cosmochim. Acta 69, 259-274. doi: 10.1016/j.gca.2004.06.024

Martin, A., and McMinn, A. (2018). Sea ice, extremophiles and life on extra-terrestrial ocean worlds. Int. J. Astrobiol. 17, 1-16. doi: $10.1017 / S 1473550416000483$

Mastrogiuseppe, M., Poggiali, V., Hayes, A., Lorenz, R., Lunine, J., Picardi, G., et al. (2014). The bathymetry of a Titan sea. Geophys. Res. Lett. 41, 1432-1437. doi: $10.1002 / 2013$ GL058618

McCord, T. B., and Castillo-Rogez, J. C. (2018). Ceres's internal evolution: The view after Dawn. Meteorit. Planet. Sci. 53, 1778-1792. doi: 10.1111/maps.13135

McCord, T. B., and Sotin, C. (2005). Ceres: evolution and current state. J. Geophys. Res. E Planets 110:E5. doi: 10.1029/2004JE002244

McCord, T. B., and Zambon, F. (2019). The surface composition of Ceres from the Dawn mission. Icarus 318, 2-13. doi: 10.1016/j.icarus.2018.03.004

McDonough, W. F., and Sun, S. S. (1995). The composition of the Earth. Chem. Geol. 120, 223-253. doi: 10.1016/0009-2541(94)00140-4 
McGrath, M. A., Hansen, C. J., and Hendrix, A. R. (2009). "Observations of Europa's Tenuous Atmosphere," in Europa, R. T. Pappalardo, W. B. McKinnon, and K. K. Khurana (Tucson, AZ: University of Arizona Press), 485-506.

McKay, C. P. (2016). Titan as the Abode of Life. Life 6:8. doi: 10.3390/life6010008

Michalski, J. R., Cuadros, J., Niles, P. B., Parnell, J., Deanne Rogers, A., and Wright, S. P. (2013). Groundwater activity on Mars and implications for a deep biosphere. Nat. Geosci. 6, 133-138. doi: 10.1038/ngeo1706

Millero, F. J., and Rabindra, N. R. (1997). A chemical equilibrium model for the carbonate system in natural waters. Croat. Chem. Acta 70, 1-38.

Mitchell, J. L., and Lora, J. M. (2016). The climate of titan. Аnnu. Rev. Earth Planet. Sci. 44, 353-380. doi: 10.1146/annurev-earth-060115012428

Mitri, G., Meriggiola, R., Hayes, A., Lefevre, A., Tobie, G., Genova, A., et al. (2014). Shape, topography, gravity anomalies and tidal deformation of Titan. Icarus 236, 169-177. doi: 10.1016/j.icarus.2014.03.018

Muñoz-Iglesias, V., Bonales, L. J., and Prieto-Ballesteros, O. (2013). pH and Salinity Evolution of Europa's Brines: Raman Spectroscopy Study of Fractional Precipitation at 1 and 300 Bar. Astrobiology 13, 693-702. doi: $10.1089 /$ ast.2012.0900

Mykytczuk, N. C. S., Foote, S. J., Omelon, C. R., Southam, G., Greer, C. W., and Whyte, L. G. (2013). Bacterial growth at $-15^{\circ} \mathrm{C}$; molecular insights from the permafrost bacterium Planococcus halocryophilus Or1. ISME J. 7, 1211-1226. doi: 10.1038/ismej.2013.8

Mykytczuk, N. C. S., Wilhelm, R. C., and Whyte, L. G. (2012). Planococcus halocryophilus sp. nov., an extreme sub-zero species from high arctic permafrost. Int. J. Syst. Evol. Microbiol. 62, 1937-1944. doi: $10.1099 /$ ijs.0.035782-0

NASA (2018). Mars Fact Sheet. Greenbelt, MD: NASA. Available at: https://nssdc. gsfc.nasa.gov/planetary/factsheet/marsfact.html (accessed September 27, 2018).

Neveu, M., and Desch, S. J. (2015). Geochemistry, thermal evolution, and cryovolcanism on Ceres with a muddy ice mantle. Geophys. Res. Lett. 42, 10197-10206. doi: 10.1002/2015GL066375

Nicholson, W. L., and Schuerger, A. C. (2005). Bacillus subtilis spore survival and expression of germination-induced bioluminescence after prolonged incubation under simulated mars atmospheric pressure and composition: implications for planetary protection and Lithopanspermia. Astrobiology 5, 536-544. doi: 10.1089/ast.2005.5.536

Noell, A. C., Ely, T., Bolser, D. K., Darrach, H., Hodyss, R., Johnson, P. V., et al. (2015). Spectroscopy and Viability of Bacillus subtilis Spores after Ultraviolet Irradiation: Implications for the Detection of Potential Bacterial Life on Europa. Astrobiology 15, 20-31. doi: 10.1089/ast.2014.1169

Norman, L. H. (2011). Is there life on ... Titan? Astron. Geophys. 52, 39-31. doi: 10.1111/j.1468-4004.2011.52139.x

Oremland, R., Kulp, T., Blum, J., Hoeft, S., Baesman, S., Miller, L., et al. (2005). A microbial arsenic cycle in a salt-saturated, extreme environment. Science 308, 1305-1308. doi: 10.1126/science. 1110832

Pasek, M. A., and Greenberg, R. (2012). Acidification of Europa's Subsurface Ocean as a Consequence of Oxidant Delivery. Astrobiology 12, 151-159. doi: $10.1089 /$ ast.2011.0666

Pavlov, A., Cheptsov, V., Tsurkov, D., Lomasov, V., Frolov, D., Vasiliev, G., et al. (2018). Survival of Radioresistant Bacteria on Europa's Surface after Pulse Ejection of Subsurface Ocean Water. Geosciences 9:9. doi: 10.3390/geosciences 9010009

Postberg, F., Kempf, S., Schmidt, J., Brilliantov, N., Beinsen, A., Abel, B., et al. (2009). Sodium salts in E-ring ice grains from an ocean below the surface of Enceladus. Nature 459, 1098-1101. doi: 10.1038/nature08046

Postberg, F., Khawaja, N., Abel, B., Choblet, G., Glein, C. R., Gudipati, M. S., et al. (2018). Macromolecular organic compounds from the depths of Enceladus. Nature 558, 564-568. doi: 10.1038/s41586-018-0246-4

Schleper, C., Puehler, G., Holz, I., Gambacorta, A., Janekovic, D., Santarius, U., et al. (1995). Picrophilus gen. nov., fam. nov.: a novel aerobic, heterotrophic, thermoacidophilic genus and family comprising archaea capable of growth around pH 0. J. Bacteriol. 177, 7050-7059. doi: 10.1128/jb.177.24.7050-7059.1995

Schleper, C., Puhler, G., Klenk, H.-P., and Zillig, W. (1996). Picrophilus oshimae and Picrophilus torridus fam. nov., gen. nov., sp. nov., two species of hyperacidophilic, thermophilic, heterotrophic, aerobic archaea. Int. J. 46, 814-816. doi: 10.1099/00207713-46-3-814
Schulze-Makuch, D., Grinspoon, D. H., Abbas, O., Irwin, L. N., and Bullock, M. A. (2004). A sulfur-based survival strategy for putative phototrophic life in the venusian atmosphere. Astrobiology 4, 11-18. doi: 10.1089/153110704773600203

Sinha, N., Nepal, S., Kral, T., and Kumar, P. (2017). Survivability and growth kinetics of methanogenic archaea at various $\mathrm{pHs}$ and pressures: implications for deep subsurface life on Mars. Planet. Space Sci. 136, 15-24. doi: $10.1016 /$ j.pss.2016.11.012

Smith, D. J., Schuerger, A. C., Davidson, M. M., Pacala, S. W., Bakermans, C., and Onstott, T. C. (2009). Survivability of Psychrobacter cryohalolentis K5 under simulated martian surface conditions. Astrobiology 9, 221-228. doi: 10.1089/ast.2007.0231

Soderlund, K. M., Schmidt, B. E., Wicht, J., and Blankenship, D. D. (2014). Oceandriven heating of Europa's icy shell at low latitudes. Nat. Geosci. 7, 16-19. doi: 10.1038/ngeo2021

Sohl, F., Solomonidou, A., Wagner, F. W., Coustenis, A., Hussmann, H., and Schulze-Makuch, D. (2014). Structural and tidal models of Titan and inferences on cryovolcanism. J. Geophys. Res. Planets 119, 1013-1036. doi: 10.1002/2013JE004512

Spencer, J. R., Tamppari, L. K., Martin, T. Z., and Travis, L. D. (1999). Temperatures on Europa from Galileo photopolarimeter-radiometer: nighttime thermal anomalies. Science 284, 1514-1516. doi: 10.1126/science.284.5419.1514

Suzuki, S., Kuenen, J. G., Schipper, K., Van Der Velde, S., Ishii, S., Wu, A., et al. (2014). Physiological and genomic features of highly alkaliphilic hydrogen-utilizing Betaproteobacteria from a continental serpentinizing site. Nat. Commun. 5:3900. doi: 10.1038/ncomms4900

Takai, K., Nakamura, K., Toki, T., Tsunogai, U., Miyazaki, M., Miyazaki, J., et al. (2008). Cell proliferation at $122 \mathrm{C}$ and isotopically heavy CH4 production by a hyperthermophilic methanogen under high-pressure cultivation. Proc. Natl. Acad. Sci. U.S.A. 105, 10949-10954. doi: 10.1073/pnas.07123 34105

Taubner, R. S., Pappenreiter, P., Zwicker, J., Smrzka, D., Pruckner, C., Kolar, P., et al. (2018). Biological methane production under putative Enceladus-like conditions. Nat. Commun. 9:748. doi: 10.1038/s41467-018-02 876-y

Teolis, B. D., Wyrick, D. Y., Bouquet, A., Magee, B. A., and Waite, J. H. (2017). Plume and surface feature structure and compositional effects on Europa's global exosphere: preliminary Europa mission predictions. Icarus 284, 18-29. doi: 10.1016/j.icarus.2016.10.027

Travis, B. J., Palguta, J., and Schubert, G. (2012). A whole-moon thermal history model of Europa: impact of hydrothermal circulation and salt transport. Icarus 218, 1006-1019. doi: 10.1016/j.icarus.2012.02.008

Vance, S. D., Hand, K. P., and Pappalardo, R. T. (2016). Geophysical controls of chemical disequilibria in Europa. Geophys. Res. Lett. 43, 4871-4879. doi: 10.1002/2016GL068547.Received

Varnes, E. S., Jakosky, B. M., and McCollom, T. M. (2003). Biological potential of Martian hydrothermal systems. Astrobiology 3, 407-414. doi: $10.1089 / 153110703769016479$

Villarreal, M. N., Russell, C. T., Luhmann, J. G., Thompson, W. T., Prettyman, T. H., A'Hearn, M. F., et al. (2017). The dependence of the cerean exosphere on solar energetic particle events. Astrophys. J. 838:L8. doi: $10.3847 / 2041-8213 /$ aa66cd

Vu, T. H., Hodyss, R., Johnson, P. V., and Choukroun, M. (2017). Preferential formation of sodium salts from frozen sodium-ammonium-chloride-carbonate brines - Implications for Ceres' bright spots. Planet. Space Sci. 141, 73-77. doi: 10.1016/j.pss.2017.04.014

Waite, J. H., Lewis, W. S., Magee, B. A., Lunine, J. I., McKinnon, W. B., Glein, C. R., et al. (2009). Liquid water on Enceladus from observations of ammonia and40Ar in the plume. Nature 460, 487-490. doi: 10.1038/nature 08153

Wayne, R. P. (2000). Chemistry of Atmospheres, 3rd Edn. Oxford: Clarendon Press. Wordsworth, R. (2016). The climate of early mars. Annu. Rev. Earth Planet. Sci. 44, 381-408. doi: 10.1146/annurev-earth-060115-012355

Zhu, P., Manucharyan, G. E., Thompson, A. F., Goodman, J. C., and Vance, S. D. (2017). The influence of meridional ice transport on Europa's ocean stratification and heat content. Geophys. Res. Lett. 44, 5969-5977. doi: 10.1002/2017GL072996

Zolotov, M. Y. (2009). On the composition and differentiation of Ceres. Icarus 204, 183-193. doi: 10.1016/j.icarus.2009.06.011 
Zolotov, M. Y. (2017). Aqueous origins of bright salt deposits on Ceres. Icarus 296, 289-304. doi: 10.1016/j.icarus.2017.06.018

Zolotov, M. Y., and Kargel, J. S. (2009). "On the chemical composition of Europa's icy shell, ocean, and underlying rocks," in Europa, eds R. T. Pappalardo, W. B. McKinnon, and K. Khurana (Tucson, AZ: University of Arizona Press), 431.

Zolotov, M. Y., Tobie, G., Postberg, F., Magee, B., Waite, J. H., and Esposito, L. (2011). Chemical and phase composition of Enceladus: insights from Cassini data. EPSC Abstracts 6:EPSC-DPS2011-1330. doi: 10.1029/2011GL047415
Copyright (c) 2019 Merino, Aronson, Bojanova, Feyhl-Buska, Wong, Zhang and Giovannelli. This is an open-access article distributed under the terms of the Creative Commons Attribution License (CC BY). The use, distribution or reproduction in other forums is permitted, provided the original author(s) and the copyright owner(s) are credited and that the original publication in this journal is cited, in accordance with accepted academic practice. No use, distribution or reproduction is permitted which does not comply with these terms. 